Viewpoint

\title{
Suicide attempts and clinical correlates in patients with bipolar I vs II disorder
}

Suicidal behaviours are extremely common amongst individuals suffering from bipolar disorder (BD), being responsible for a considerable disease burden for patients and caregivers [1]. Indeed, patients with BD type I (BDI) vs II (BDII) have shown multiple differences in terms of socio-demographic and clinical features in several studies, even though only few reports focused on clinical characteristics potentially differentiating BDI vs BDII patients with a previous suicide attempt (PSA) [2,3]. In this respect, the different prevalence of PSA between BDI and BDII is still debated, with a recent systematic review of 101 previous studies reporting a similar PSA rate in the two subtypes [4]. Nonetheless, a recent study conducted on $494 \mathrm{BD}$ outpatients showed PSA rate was approximately twice as high in BDI patients with compared to without lifetime eating disorder and in BDII patients with compared to without childhood BD onset. Moreover, in the same study, PSA rates were significantly higher in BDI patients with current antidepressant use, in BDII female patients, and in BDII subjects with a lifetime history of psychosis [2].

In light of the limited investigation in the field, particularly in relation to Italian patients, and in order to better characterize clinical correlates of BDI vs II patients with PSA, we conducted the present study on a large sample of Italian bipolar patients, hypothesizing the existence of distinctive socio-demographic and clinical characteristics across groups.

From an original sample of 362 bipolar in- and out-patients referred to the University Clinic and related community services of the Department of Psychiatry, Fondazione IRCCS Ca' Granda Policlinico of Milan, 94 subjects with a lifetime PSA were identified for the research purpose. Detailed diagnostic inclusion criteria and collected variables have been specified elsewhere [5]. With respect to PSA, lifetime number, methods and severity of attempts were assessed by clinicians.

Collected variables were compared between BDI and BDII patients with PSA (BDI-PSA and BDII-PSA). Continuous sociodemographic and clinical variables were analysed using Student's $t$-test (or Mann-Whitney $U$ test for non-parametric variables), whereas Chi-Squared tests with post-hoc test (Bonferroni) were used for categorical parameters. The Statistical Package for the Social Sciences for Windows Software, version 24, was used for analyses, and the threshold for statistical significance was set at a two-tailed-value of 0.05 .
In our sample, BDI diagnosis was approximately three-fold more common than BDII (BDI: 71 patients vs BDII: 23 patients), with statistical analysis showing no significant difference in terms of socio-demographic variables. No difference emerged between PSA rate in relation to BD subtypes (BDI-PSA patients $26.5 \%$ vs BDIIPSA 25\%). Moreover, lifetime number, methods and severity of PSA were similar between BDI-PSA vs BDII-PSA subjects.

In relation to clinical variables, BDI-PSA vs BDII-PSA patients showed a significantly higher number of psychiatric hospitalizations $(5.9 \pm 9.4$ vs $2.7 \pm 0.9, Z=-3.8, p<0.001)$, whereas a similar rate of lifetime involuntary commitments was observed.

The two subgroups were similar in terms of overall psychiatric comorbidity rates, even though substance use disorder (SUD) was more frequently observed in BDI-PSA compared to BDII-PSA (27.1\% vs $\left.4.5 \%, \chi^{2}=1.6, \mathrm{p}<0.05\right)$. No further differences emerged in relation to medical or other psychiatric comorbidities.

A significantly higher lifetime rate of psychotic symptoms (70.4\% vs 9.1\%, $\chi 2=25.6, p<0.001$ ) was found in BDI-PSA vs BDIIPSA patients. Additionally, BDI-PSA patients showed a lower global assessment of functioning (GAF) score $(60.3 \pm 13.8$ vs $87.7 \pm 12.0$, $\mathrm{F}=1.1, \mathrm{p}<0.05$ ) compared with BDII-PSA patients.

In relation to episode polarity at the onset, a depressive polarity was more frequently associated with BDII-PSA in comparison with BDI-PSA patients ( $87 \%$ vs $60.3 \%, \chi 2=5.4, p<0.001$ ) and, on the other hand, BDI-PSA patients more frequently had an elevated mood episode. Moreover, a depressive polarity of most recent episode was more frequently observed in BDII-PSA vs BDI-PSA patients (95.5\% vs 33.8\%, $\chi 2=24.9, \mathrm{p}<0.001$ ). Additionally, BDIIPSA vs BDI-PSA patients had a significantly higher frequency of lifeevents associated with the onset of BD (78.3\% vs $52.5 \%, \chi^{2}=4.5$, $\mathrm{p}<0.05$ ). Finally, BDII-PSA patients showed a longer duration of untreated illness (DUI) compared to BDI-PSA ones, without reaching the statistically significant threshold $(8.5 \pm 10.1$ vs $3.9 \pm 7.4$ years, $\mathrm{F}=7.8, \mathrm{p}=0.53$ ).

With respect to treatment, current antidepressants use was more commonly observed in BDII-PSA subjects, compared to BDIPSA ones (59.1\% vs 23.5\%, $\chi 2=9.6, \mathrm{p}<0.05$ ). On the other hand, similar rates of antipsychotics and mood stabilizers use between the two subgroups were found. Fig. 1 shows categorical variables with statistically significant differences between BDI and BDII patients with a PSA. 


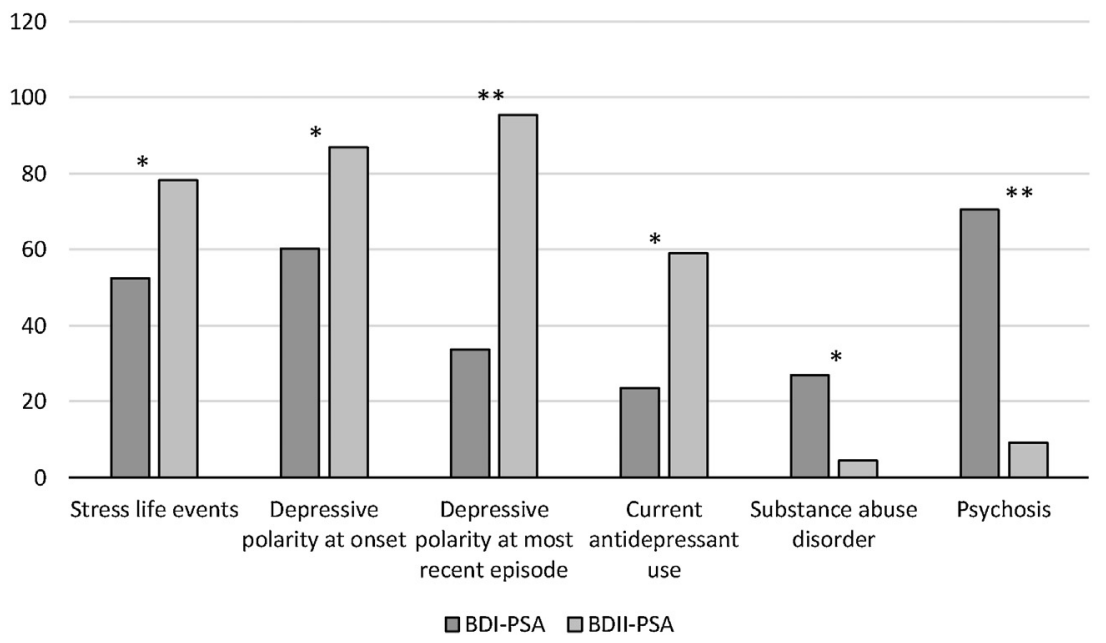

Fig. 1. Statistically significant differences between BDI and BDII patients with a PSA, categorical variables.

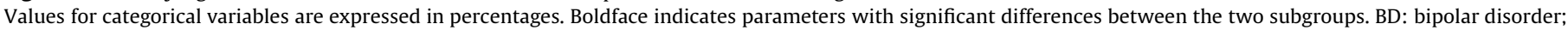
PSA: previous suicide attempt. Statistics: ${ }^{*} \mathrm{p}<.05,{ }^{* *} \mathrm{p}<.001$.

In light of existing evidence showing similar lifetime rates of PSA in bipolar disorders - as confirmed by the present study - we contend for a differentiation of suicide attempters with BD on the basis of specific clinical correlates. In fact, our finding showing a similar PSA rate between BD I and II patients is consistent with most of prior studies, as reported in collaborative reports of our [5] and other groups [3,4]. Nonetheless, a few studies reported mixed results in terms of PSA rates across BD subtypes, with a higher PSA rate in BDI [6] or in BDII patients [7]. These differences may be due to several factors, including different sample recruitment and methodological approaches in collecting patients' variables, geographic differences across samples, and the inconsistent distribution of BDI and BDII patients across analysed samples.

In the present study, BDII-PSA patients showed a lower lifetime rate of psychotic symptoms. Additionally, BDII-PSA patients were more frequently receiving antidepressants, while BDI subjects did not show any difference in terms of type or number of psychotropic compounds. We explained this result considering the traditionally minor presence of psychotic symptoms and the more frequent use of antidepressants [8] in the BDII population, rather than a causeeffect relationship between such variables and PSA.

BDI-PSA vs BDII-PSA patients seemed to show overall worse clinical characteristics. In particular, a higher number of psychiatric hospitalizations, a higher rate of psychotic symptoms, a lower GAF score and a higher rate of comorbid SUD were significantly more present in BDI vs BDII subjects with PSA. On the other hand, depressive polarity at most recent episode and at onset was found to be significantly more present in BDII-PSA patients. As consistently reported, depressive polarity at onset is a variable associated with a poor clinical outcome in BD and, in particular, with a higher suicidal risk [9]. In addition, in our sample, BDII vs BDI subjects with a PSA showed a longer DUI. This variable has been, in turn, associated with worse prognosis and outcome, as indicated by a previous study of our group showing an association between a longer DUI and a higher suicidal risk in BD [10]. Along with literature reports showing that individuals with BDII attempted suicide with more violent and lethal methods than BDI patients [3], results from the present study seem to confirm the notion that BDII patients with PSA suffer from an equally severe form of illness compared with BDI patients.

With respect to substance abuse, a higher rate of comorbid SUD in BDI-PSA patients emerged in the present report. Indeed, Goffin and colleagues showed that SUD was associated with PSA in both bipolar subtypes, even though with a stronger statistical significance in BDI [2]. Moreover, the above-mentioned study reported an additional link, with BDI-PSA patients more frequently reporting comorbid anxiety and eating disorders compared to BDII ones [2].

From our viewpoint, the aforementioned studies support the view of BDI and BDII as conditions with distinct socio-demographic and clinical profiles that should always be separately analysed in clinical reports. In fact, some characteristics might be peculiar to one or to the other subtype and may be responsible for the heterogeneous results reported when they are considered as a unique entity. Apart from research purposes, considering BDI and BDII separately has relevant clinical implications. In light of reported findings, for instance, clinicians should stay alert for possible increased risk of suicide attempts in BDI patients with an increased number of psychiatric hospitalizations, comorbid SUD, psychotic symptoms, and a low GAF score and, on the other side, in BDII patients with stressing life-events at onset, depressive first and most recent episode polarity, and current antidepressant use.

In this light, we believe that current risk assessment and interventions targeted to reduce risk factors must represent a priority in the clinical management and treatment of bipolar patients, with the aim to reduce suicide-related morbidity and mortality.

For a correct interpretation of the aforementioned results, the following methodological limitations should be taken into consideration. First, BDI patients were numerically more represented: this difference may have potentially affected reported data, even though diagnostic subgroups did not differ significantly for age, gender, and education. Due to the nature of the study, all collected variables were obtained retrospectively and, accordingly, susceptible to recall bias. In addition, the severity of PSA was assessed by means of a descriptive criterion and not through a specific standardized scale. Finally, due to the nature of the study, we could not ascertain the potential role of certain clinical characteristics found to be associated with a specific BD subtype, independently from PSA. Therefore, further multicenter studies with larger samples and longitudinal follow-up are warranted to confirm our findings.

\section{References}

[1] Pompili M., Gonda X, Serafini G, Innamorati M, Sher L, Amore M, et al Epidemiology of suicide in bipolar disorders: a systematic review of the literature. Bipolar Disord 2013;15:457-90, doi:http://dx.doi.org/10.1111/ bdi.12087. 
[2] Goffin KC, Dell'Osso B, Miller S, Wang PW, Holtzman JN, Hooshmand F, et al. Different characteristics associated with suicide attempts among bipolar I versus bipolar II disorder patients. J Psychiatr Res 2016;76:94-100, doi:http:// dx.doi.org/10.1016/j.jpsychires.2016.02.006.

[3] Novick DM, Swartz HA, Frank E. Suicide attempts in bipolar I and bipolar II disorder: a review and meta-analysis of the evidence. Bipolar Disord 2010;12:1-9, doi:http://dx.doi.org/10.1111/j.1399-5618.2009.00786.x.

[4] Tondo L, Pompili M, Forte A, Baldessarini RJ. Suicide attempts in bipolar disorders: comprehensive review of 101 reports. Acta Psychiatr Scand 2016;133:174-86, doi:http://dx.doi.org/10.1111/acps.12517.

[5] Dell'Osso B, Vismara M, Dobrea C, Cremaschi L, Grancini B, A rici C, et al. Clinica characterization of Italian suicide attempters with bipolar disorder. CNS Spectr 2017, doi:http://dx.doi.org/10.1017/S1092852917000384.

[6] Bega S, Schaffer A, Goldstein B, Levitt A. Differentiating between bipolar disorder types I and II: results from the national epidemiologic survey on alcohol and related conditions (NESARC). J Affect Disord 2012;138:46-53, doi: http://dx.doi.org/10.1016/j.jad.2011.12.032.

[7] Baek JH, Park DY, Choi J, Kim JS, Choi JS, Ha K, et al. Differences between bipolar I and bipolar II disorders in clinical features, comorbidity, and family history. J Affect Disord 2011;131:59-67, doi:http://dx.doi.org/10.1016/j.jad. 2010.11.020.

[8] Baldessarini RJ, Leahy L, Arcona S, Gause D, Zhang W, Hennen J. Patterns of psychotropic drug prescription for U.S. patients with diagnoses of bipolar disorders. Psychiatr Serv 2007;58:85-91, doi:http://dx.doi.org/10.1176/ ps.2007.58.1.85.

[9] Cremaschi L, Dell'Osso B, Vismara M, Dobrea C, Buoli M, Ketter TA, et al. Onset polarity in bipolar disorder: a strong association between first depressive episode and suicide attempts. J Affect Disord 2017;209:182-7, doi:http://dx. doi.org/10.1016/j.jad.2016.11.043.

[10] Altamura AC, Dell'Osso B, Berlin HA, Buoli M, Bassetti R, Mundo E. Duration of untreated illness and suicide in bipolar disorder: a naturalistic study. Eur Arch Psychiatry Clin Neurosci 2010;260:385-91, doi:http://dx.doi.org/10.1007/ s00406-009-0085-2.
Chiara Arici

Vera de Carlo

University of Milan, Department of Mental Health, Fondazione IRCCS Ca' Granda Policlinico, Milan, Italy

Shefali Miller

Terence A. Ketter

Department of Psychiatry and Behavioral Sciences, Bipolar Disorders Clinic, Stanford University, CA, USA

A. Carlo Altamura

University of Milan, Department of Mental Health, Fondazione IRCCS Ca' Granda Policlinico, Milan, Italy

Bernardo Dell'Osso ${ }^{\mathrm{a}, \mathrm{b}, \mathrm{c}, *}$

${ }^{a}$ University of Milan, Department of Mental Health, Fondazione IRCCS Ca' Granda Policlinico, Milan, Italy

${ }^{b}$ Department of Psychiatry and Behavioral Sciences, Bipolar Disorders Clinic, Stanford University, CA, USA

c"Aldo Ravelli" Center for Neurotechnology and Brain Therapeutic, University of Milan, Milan, Italy

* Corresponding author at: Department of Psychiatry, University of Milan, Fondazione IRCCS Ca' Granda Policlinico, Via F. Sforza, 35, 20122, Milan, Italy. E-mail address: bernardo.dellosso@unimi.it (B. Dell’Osso).

Received 27 March 2018

Available online 17 May 2018 